\title{
Genome-wide association study for crown rust (Puccinia coronata f. sp. avenae) and powdery mildew (Blumeria graminis f. sp. avenae) resistance in an oat (Avena sativa) collection of commercial varieties and landraces
}

\author{
Gracia Montilla-Bascón ${ }^{1+}$, Nicolas Rispail ${ }^{1+}$, Javier Sánchez-Martín ${ }^{1}$, Diego Rubiales $^{1}$, Luis A. J. Mur ${ }^{2}$, \\ Tim Langdon ${ }^{2}$, Catherine J. Howarth ${ }^{2}$ and Elena Prats ${ }^{1}$ *
}

I Institute for Sustainable Agriculture - Consejo Superior de Investigaciones Científicas, Córdoba, Spain

2 Institute of Biological, Environmental and Rural Sciences, University of Aberystwyth, Aberystwyth, UK

\section{Edited by:}

Jaime Prohens, Universitat Politècnica de València, Spain

Reviewed by:

Soren K. Rasmussen, University of Copenhagen, Denmark

Fernando Martinez, University of Seville, Spain

Jason Wallace, Cornell University, USA

\section{*Correspondence:}

Elena Prats, Institute for Sustainable Agriculture - Consejo Superior de Investigaciones Científicas, Apartado 4084, E-14080 Córdoba, Spain e-mail: elena.prats@ias.csic.es

${ }^{t}$ These authors have contributed equally to this work.
Diseases caused by crown rust (Puccinia coronata f. sp. avenae) and powdery mildew (Blumeria graminis f. sp. avenae) are among the most important constraints for the oat crop. Breeding for resistance is one of the most effective, economical, and environmentally friendly means to control these diseases. The purpose of this work was to identify elite alleles for rust and powdery mildew resistance in oat by association mapping to aid selection of resistant plants. To this aim, 177 oat accessions including white and red oat cultivars and landraces were evaluated for disease resistance and further genotyped with 31 simple sequence repeat and 15,000 Diversity Arrays Technology (DArT) markers to reveal association with disease resistance traits. After data curation, 1712 polymorphic markers were considered for association analysis. Principal component analysis and a Bayesian clustering approach were applied to infer population structure. Five different general and mixed linear models accounting for population structure and/or kinship corrections and two different statistical tests were carried out to reduce false positive. Five markers, two of them highly significant in all models tested were associated with rust resistance. No strong association between any marker and powdery mildew resistance at the seedling stage was identified. However, one DArT sequence, oPt-5014, was strongly associated with powdery mildew resistance in adult plants. Overall, the markers showing the strongest association in this study provide ideal candidates for further studies and future inclusion in strategies of marker-assisted selection.

Keywords: association analysis, drought, oat, powdery mildew, resistance, crown rust

\section{INTRODUCTION}

Oat is a grain crop of Mediterranean origin used for both human food and animal feed as well as a green or conserved fodder and, more recently, as a winter cover crop in no-till rotations (Stevens et al., 2004). Avena sativa L. including both white and red oat (formerly A. byzantina K. Koch) is the main cultivated oat. Several pathogenic fungi can infect oats and drastically reduce its yield including biotrophic pathogens such as powdery mildew (Blumeria graminis f. sp. avenae Em. Marchal) and crown rust (Puccinia coronataf. sp. avenae Eriks). These fungi have very efficient spreading mechanisms, hampering their control by crop management measures such as rotation and the use of resistant varieties is one of the best control alternatives (Stevens et al., 2004).

Genetic markers have proved useful for the identification of quantitative trait loci (QTL) associated with important agronomic traits using a number of experimental bi-parental oat populations. Examples include vernalization response, flowering, and heading date (Maloney etal., 2011), quality traits, including seed tocopherol (Jackson et al., 2008), groat protein and oil content (Zhu et al., 2004; Hizbai et al., 2012) and resistance to stresses including winter field survival (Maloney et al., 2011), Fusarium resistance (He et al., 2013), powdery mildew resistance (Yu and Herrmann, 2006), and crown rust resistance (Wight et al., 2004; Portyanko et al., 2005; Jackson et al., 2010). However, there are often limitations in the use of such QTL in marker-assisted selection (MAS) as the parental genotypes used in these studies are often not representative of the germplasm pool that is actively used in breeding programs and markers linked to QTL are not always transferable to other genetic backgrounds (Snowdon and Friedt, 2004).

Association analysis is an alternative approach that overcomes many of the limitations of conventional QTL mapping and has received increasing attention from plant geneticists during the last few years (Kraakman et al., 2004; Gupta et al., 2005; Breseghello and Sorrells, 2006; Stracke etal., 2009) following its success in dissecting human diseases (Klein et al., 2005; Cordell et al., 2013; Lee et al., 2013). Association analysis relies on unrelated individuals to create population-wide marker-phenotype associations (Jannink et al., 2001) and is based on linkage disequilibrium, defined as the non-random association of alleles at two loci (Falconer and MacKay, 1996). Linkage disequilibrium 
among loci is a complex phenomenon, since it is affected by mutation history, population structure, admixture among populations, natural and artificial selection (including breeding), genetic drift, and the organism's own reproductive biology (FlintGarcia et al., 2003; Newell et al., 2011). Association analysis utilizes historic patterns of recombination that have occurred within a sample of individuals to detect correlations between genotypes and phenotypes within these individuals (Zondervan and Cardon, 2004).

In recent years, genome wide association studies (GWAS) have identified marker-trait associations for a range of agronomic traits in many crops including maize, rice, sorghum, and foxtail millet (Huang etal., 2010; Jia etal., 2013; Li et al., 2013; Morris et al., 2013). However, there are fewer reports of the use of GWAS with stress resistance traits although marker associations with disease resistance have been identified in maize, rice, and wheat (Kump et al., 2011; Gurung et al., 2014; Wang et al., 2014). In oats, only a few association analysis studies have been reported (Achleitner et al., 2008) and they have primarily focussed on grain quality traits such as beta-glucan concentration (Newell et al., 2012; Asoro et al., 2013) and none have attempted to identify marker-trait associations with some of the most important biotic constraints of this crop, namely powdery mildew and rust pathogenic fungi. In this work, we performed an association analysis in an oat collection of commercial cultivars and landraces based on simple sequence repeat (SSR) and Diversity Arrays Technology (DArT) genotyping following a detailed study of population structure and linkage disequilibrium and identified several markers associated with rust and powdery mildew resistance.

\section{MATERIALS AND METHODS PLANT MATERIAL}

For this study, a germplasm collection of landraces consisting of 141 A. sativa accessions ( 110 white and 31 red oats) kindly provided by the "Centro de Recursos Fitogenéticos," INIA, Madrid, Spain, and 36 commercial varieties supplied by the Andalusian Network of Agriculture Experimentation (RAEA) was used. Oat cultivars studied were: Ac1, Acebeda, Adamo, Aintree, Alcudia, Anchuela, Araceli, Brawi, Caleche, Cannele, Chambord, Chappline, Charming, Cobeña, Condor, Cory, Edelprinz, Flega, Fringante, Fuwi, Hammel, Kankan, Kantora, Karmela, Kassandra, Kazmina, Mirabel, Mojacar, Norly, Orblanche, Pallini, Patones, Prevision, Primula, Rappidena, and Saia. Details of the origin of all accessions and of their genetic relationship have been previously reported in Montilla-Bascón et al. (2013).

Seedlings were grown in $0.5 \mathrm{~L}$ pots filled with peat:sand (3:1) in a growth chamber with $20^{\circ} \mathrm{C}, 65 \%$ relative humidity $(\mathrm{RH})$ and under $12 \mathrm{~h}$ dark/ $12 \mathrm{~h}$ light with $250 \mu \mathrm{mol} \mathrm{m} \mathrm{m}^{-2} \mathrm{~s}^{-1}$ photon flux density supplied by high-output white fluorescent tubes.

\section{GENOTYPING AND DATA CURATION}

First leaves from 40 12-days-old seedlings were harvested, pooled together, and DNA extracted according to the method stipulated by Diversity Arrays P/L, Canberra, ACT, Australia and described by Tinker et al. (2009). SSR analysis was as previously described Montilla-Bascón et al. (2013). SSRs used were chosen for their amplification consistency and polymorphism in our oat genotypes and/or because they displayed reasonable genome coverage in a mapping population developed from the winter oat cultivars Buffalo and Tardis (C. J. Howarth personal communication). DArT marker analysis using the high density oat array $(15,000$ markers) was performed by Diversity Arrays P/L, as described in Tinker et al. (2009).

To remove possible errors and redundancies in markers that may cause false associations in GWAS, data cleaning was performed according to Miyagawa et al. (2008). Markers with $>20 \%$ missing data were removed as were those with a minor allele frequency (MAF) of less than 1\%. Markers that diverged less than 1\% across the genotypes lines were merged, thus combining markers that were in near perfect LD. Finally, inspections were performed to determine accessions that differed by less than $1 \%$ to remove any redundant accessions, however, in our study no accessions fell into this category.

\section{GENETIC DISTANCE, POPULATION STRUCTURE, AND KINSHIP}

Estimates of genetic distance were calculated according to Nei and Li (1979) parameter with Arlequin software. Population structure was inferred by the software STRUCTURE 2.3.4 (Pritchard et al., 2000) using the admixture model and the option of correlated allele frequencies between populations. Similarly, the degree of admixture alpha was inferred from the data. Each simulation included 20,000 burn-in and 100,000 iterations. Longer burn-in or MCMC did not change significantly the results. Ten independent simulations per $k$-value were run. Then, the mean estimate across runs of the log posterior probability of the data for a given $k$, were plotted to enable the determination of the $k$-value of the population. As this point is known to be difficult to determine, the $\Delta \mathrm{k}$, related to the second order rates of change of the likelihood function with respect to $k$, was also used (Evanno et al., 2005). The percentages of admixture of each accession ( $Q$ matrix) given by the software were used as cofactors in the association analyses. For trait analyses per subpopulation, an accession was assigned to a subpopulation when it showed more than $80 \%$ membership in this subpopulation (de Alencar Figueiredo et al., 2010). Principal component analysis (PCA) was also performed as an alternative method to infer the structure of the collection with the software package PAST (Hammer et al., 2001).

The kinship coefficient approach proposed by Yu et al. (2006) allows taking possible family relatedness into account and can help removing additional false positives. These coefficients ( $\mathrm{K}$ matrix) were computed with the software TASSEL 4.1.27 (Bradbury et al., 2007).

\section{LINKAGE DISEQUILIBRIUM}

Linkage disequilibrium measured as $r^{2}$ was calculated by software TASSEL 4.1.27 for each marker pair together with the significance of the parameter. $r^{2}$ was used, since it is only moderately influenced by small sample sizes and low allele frequencies (Flint-Garcia etal., 2003) and it is relevant for QTL mapping since it relates the amount of variance explained by the marker to the amount of variance generated by the associated QTL (Zhu et al., 2008). The disequilibrium matrix summarizing 
pair-wise measures of LD was also performed with the software TASSEL.

\section{PHENOTYPING}

\section{Crown rust resistance assessment}

The $P$. coronata f. sp. avenae $(\mathrm{Pca})$ isolate Co-04, previously multiplied on the susceptible cultivar Araceli, was used. The virulence of this isolate on an oat differential set collection has been described in Sánchez-Martín et al. (2012). Four independent plants per accession were grown in a growth chamber as described above and when the first leaf had fully expanded they were inoculated with urediospores mixed with pure talcum powder $(1: 1, \mathrm{w} / \mathrm{w})$ by dusting them over the plants to give approximately 30 spores $\mathrm{mm}^{-2}$ (checked by counts made from glass slides laid adjacent to leaves). After inoculation, plants were incubated for $9.5 \mathrm{~h}$ in darkness at $100 \% \mathrm{RH}$ and $18^{\circ} \mathrm{C}$, and thereafter at $20^{\circ} \mathrm{C}$ under a $12 \mathrm{~h}$ photoperiod with $250 \mu \mathrm{mol} \mathrm{m} \mathrm{m}^{-2} \mathrm{~s}^{-1}$ photon flux density. Infection frequency (IF) was determined as previously described in Prats et al. (2002). IF scores were converted into relative infection frequency (RIF) values expressed as the percentage of the susceptible reference cultivar Araceli.

\section{Powdery mildew resistance assessment}

Four independent plants per accession were grown as described above and when the first leaf was fully expanded it was inoculated using a settling tower (Lyngkjær et al., 1997) to give about 30 conidia $\mathrm{mm}^{-2}$ with one isolate of $B$. graminis f. $\mathrm{sp}$. avenae race five maintained on seedlings of oat cv. Selma, in a spore proof glasshouse. After inoculation, plants were maintained in the growth chamber for 8 days before assessment of the percentage area covered by powdery mildew on the inoculated leaf. Disease scores were converted into relative values, expressed as the percentage of the susceptible reference cultivar Selma and referred to as the relative disease severity (RDS; Rubiales et al., 1993; Martínez et al., 2007). For assessment of adult plant resistance the fifth leaves were inoculated and macroscopically assessed as above without excising the leaves from the plant.

\section{STATISTICAL ANALYSES}

For phenotype assessments the experimental design was arranged according to randomized complete block design with four independent blocks each containing the whole set of accessions randomly ordered. For ease of understanding, means of raw percentage data are presented in tables and figures. However, for statistical analysis, data recorded as percentages were transformed to arcsine square roots (transformed value $=180 / \Pi \times$ arcsine $[\sqrt{ }(\% / 100)])$ to normalize data and stabilize variances throughout the data range, and subjected to analysis of variance using SPSS software, after which residual plots were inspected to confirm that data conformed to normality. Significance of differences between means was determined by contrast analysis (Scheffe's). The percentage of variation of each trait explained by the structure was computed through multiple linear regression of the phenotypes on the percentages of admixture using $\mathrm{R}$ (Ihaka and Gentleman, 1996).

\section{ASSOCIATION ANALYSIS}

Associations between molecular markers and phenotypes were computed using the software package TASSEL 4.1.27 (Bradbury et al., 2007). Five models were used: a simple general linear model (GLM), a GLM model using the percentages of admixture of each accession ( $Q$ matrix) as cofactors to take population structure into account (GLM-Q), a GLM model using the PCAs covariates as cofactors (GLM-PCA), a GLM model using both Q matrix and PCAs covariates (GLM-Q-PCA) and a mixed linear model (MLM) using both the percentages of admixture and the kinship coefficients as cofactors ( $Q$ and K matrices). All GLM procedures tested fixed-effect models in which mean phenotypes of a given trait were predicted by the independent variables. Tests were run with 1,000 permutations allowing determination of the site-wise $p$-value for each marker, which is the probability of a greater $F$-value under the null hypothesis that the polymorphic site is independent of phenotype. All models were assessed for their ability to control for type I error by plotting the distribution of the $p$-values for the markers, where uniformly distributed $p$-values indicate proper control for type I errors (Newell et al., 2012). The Benjamini and Hochberg (1995) false discovery rate (FDR) criteria at $q=0.25$ was used to control for multiple testing (Newell et al., 2012) after estimation of the $q$-values of each $p$-values with the module QVALUE (Storey, 2002) in the $\mathrm{R}$ v2.15.2 package.

\section{SEOUENCE HOMOLOGY}

As many of the DArT markers used here have been previously sequenced (Tinker et al., 2009), the NCBI non-redundant protein database (database released on 11 January 2015) was searched using the function BlastX of the BLAST algorithm (Altschul etal., 1990) implemented in the NCBI webserver (http://blast.ncbi.nlm.nih.gov/Blast.cgi) to further characterize the most significant markers identified.

\section{RESULTS}

\section{DATA CURATION}

Of the initial 15,000 DArT markers assessed, 1,587 showed polymorphism in the oat collection. In addition 499 SSR alleles were also polymorphic. From the total 2086 polymorphic markers, 11 markers that showed a call rate lower than $80 \%$ and 56 markers that showed a MAF $<0.01$ were removed. A total of 476 redundant markers were also merged in 169 groups representing these markers. Following data curation a total of 1,712 markers were used for association purposes in the oat collection of 177 white and red commercial varieties and landraces.

\section{STRUCTURE OF THE POPULATION}

A previous genetic diversity study of the oat collection with only SSR markers revealed a structure of four subpopulations (Montilla-Bascón et al., 2013). In the present study the number of markers was increased to more than 1,500 and STRUCTURE software indicated the same number of subpopulations (Figure 1). Indeed, the correlation between SSR and DArT+SSR results was high with a correlation coefficient of $0.84(p<0.001)$. However, slight modifications of the genotype-cluster assignation and the corresponding percentage of admixture were observed. According to both analyses $\sim 30 \%$ of the accessions showed 


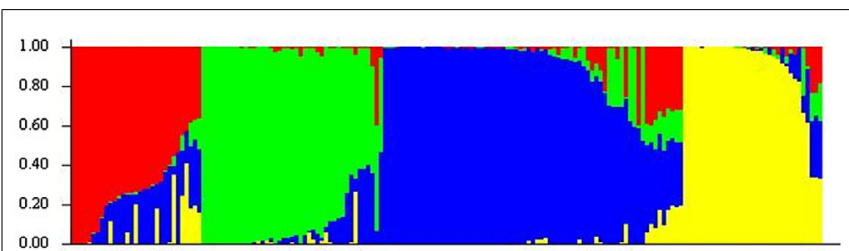

FIGURE 1 | Estimated population structure of oat genotypes according to STRUCTURE software. Each individual is represented by a thin vertical segment, which can be partitioned into four colored segments that represent the individual estimated membership to the four clusters.

less than $80 \%$ of membership for a particular cluster. The differences of genotype-cluster assignation were always related to these accessions and when they were discarded from the analysis the correlation coefficient increased up to 1. According to STRUCTURE, subpopulation 1 showed the highest degree of admixture with $75.6 \%$ of the genotypes with less than $80 \%$ of membership to this subpopulation followed by subpopulations 3 and 4 with $18 \%$ of genotypes with less than $80 \%$ of membership in these groups. Subpopulation 2 in which only $15 \%$ of genotypes showed less than $80 \%$ membership to the corresponding subpopulation was the subpopulation with lowest admixture.

Multivariate analysis based on PCA also revealed a separation of four subpopulations which indicates a high consistency of the data (Figure 2). Cluster analysis was implemented on the first four principal components cumulatively explaining $\sim 50 \%$ of the variation with $23.3,13.8,8.11$, and $4.5 \%$ for each of the components, respectively. Although separation between clusters was clear, some accessions were not part of the clusters but formed links between them (Figure 2). The number of lines in each cluster ranged from 33 to 64 . The first cluster included mainly the white commercial

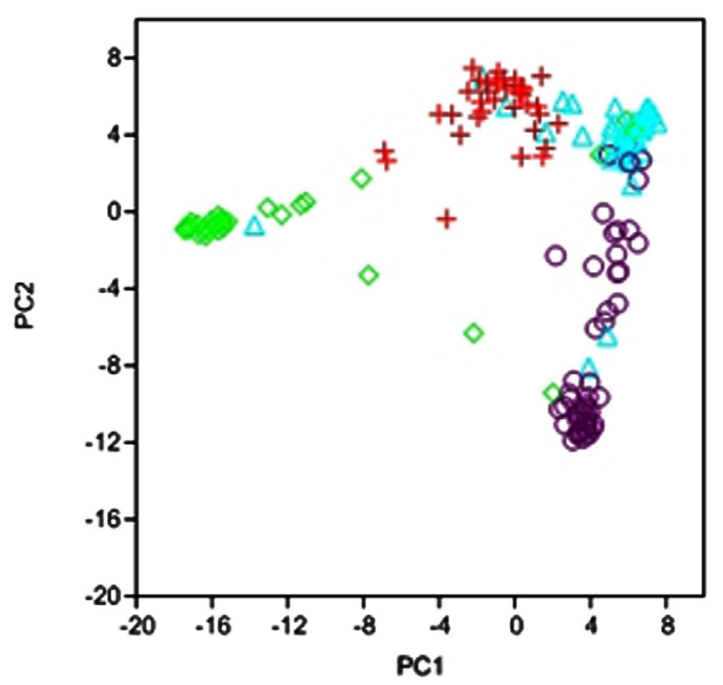

FIGURE 2 | Scatterplot of Principal Component Analysis scores of components 1 and 2 based on 1712 DArT and SSR markers used in this study. Represented are the genotypes belonging to cluster 1 (red), cluster 2 (green), cluster 3 (violet), and cluster 4 (blue). varieties, cluster 2 the red oats, cluster 3 the white oat landraces characteristic to high altitude locations, and cluster 4 white oat landraces more adapted to low altitude locations.

\section{CLUSTER RELATIONSHIPS}

As previously stated, PCA showed a separation between clusters but also a clear pair-wise relationship between clusters. Quantitative results for genetic distance (according to Nei's parameter) between clusters are shown in Table 1. Cluster 2 comprising the red oats was by far the most distant from all other clusters, with an average distance of 180 whereas the two white oat landrace clusters were the most closely related groups with an average distance of 66 . Clusters 1 and 4 corresponding to the white oat landraces adapted to low altitude and the commercial varieties, respectively were also closely related with a distance of 75 (Table 1). These relationships between clusters were in agreement with those depicted by the PCA scatter plot (Figure 2). These results suggest that clustering was also efficient in separating the oat types for the germplasm used in this study.

\section{LINKAGE DISEOUILIBRIUM}

Identification of disequilibrium between markers is highly useful since it may condition the strength of the association study. Since physical map distances between markers were not available, LD was represented by the disequilibrium matrix visualizing the linear arrangement of LD between polymorphic sites, represented by $r^{2}$, and the probability (Flint-Garcia et al., 2003; Gaut and Long, 2003; Figure 3). A total of 507,042 pairs of markers showed a significant $\mathrm{LD}$ value with an average $p=0.004$. From these, 277,920 pairs of markers showed an $r^{2}<0.1$ chosen here as nominal level, according to the studies performed by Newell et al. (2011) in oat. LD of each cluster showed similar values with the exception of cluster 2 that showed a slightly higher LD, probably reflecting the low number of individuals of this cluster of red oats.

\section{PHENOTYPIC DATA}

Both traits followed a normal distribution with accessions ranging from highly resistant to highly susceptible (Figure 4). Means of the 177 accessions assigned to the four subpopulations for the different traits, excluding the admixed accessions were compared (Table 2). Significant differences between subpopulations were observed for all traits. Thus, subpopulation 4 had a significantly lower RIF after rust inoculation than the others $(p<0.005)$ and showed a high resistant response. Subpopulation 2 had lower RDS to powdery mildew than the others ( $p<0.001$; Table 2$)$. Powdery mildew was the trait most affected by population structure

Table 1 | Population average pair-wise genetic distance according to Nei's parameter of pair-wise difference.

\begin{tabular}{lccc}
\hline & C1 & C2 & C3 \\
\hline C2 & 150 & & \\
C3 & 125 & 205 & \\
C4 & 75 & 185 & 66
\end{tabular}




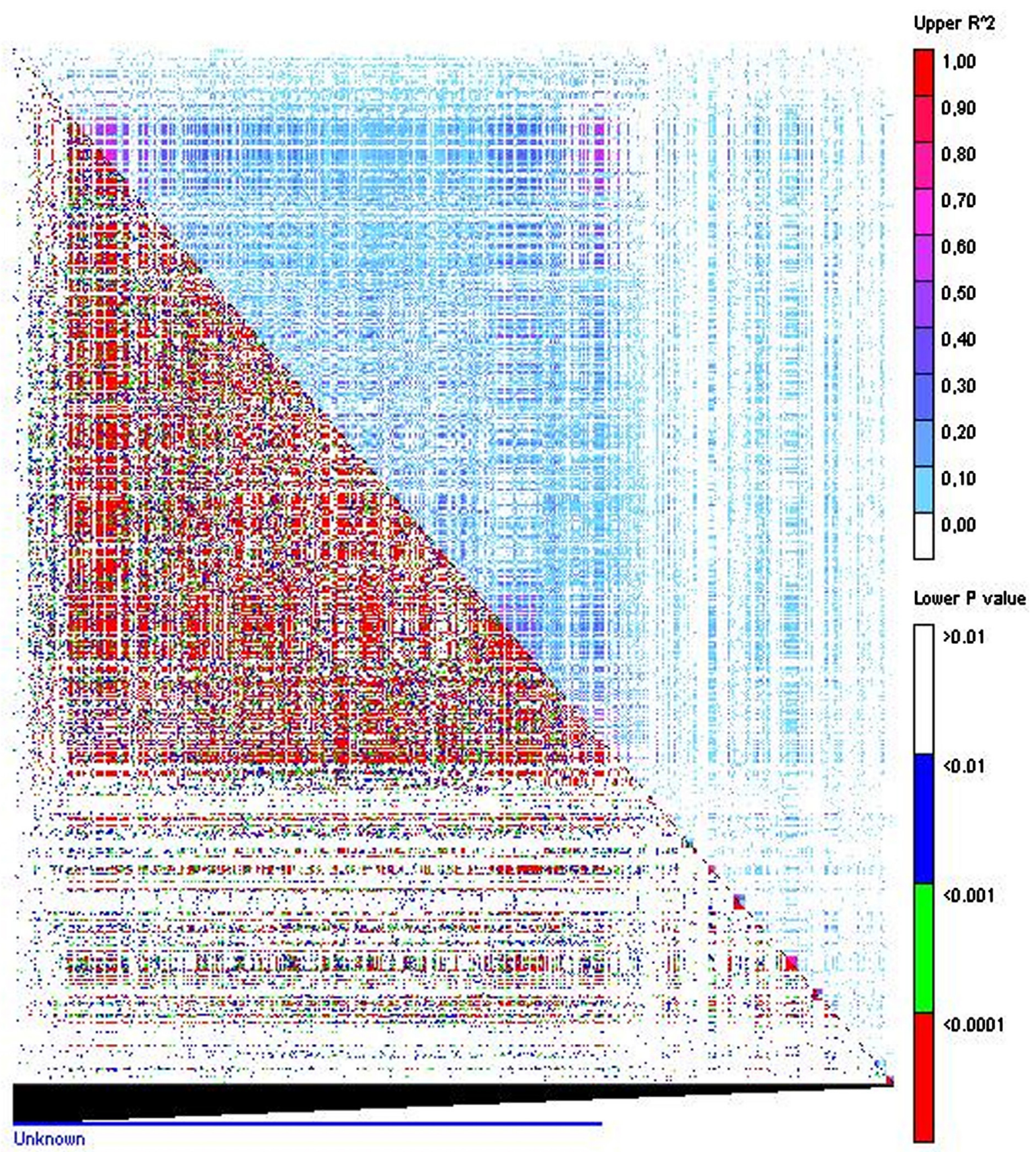

FIGURE 3 | Linkage disequilibrium matrix. Pair-wise LD values of polymorphic sites displaying $r^{2}$ above the diagonal and the corresponding p-values from rapid 1000 shuffle permutation test below the diagonal. Each cell represent the comparison of two pairs of marker sites with the color codes for the presence of significant LD. Colored bar code for the significance threshold levels in both diagonals is shown. although the proportion of variance explained by population structure remained under $4 \%$ (Table 3).

\section{ASSOCIATION ANALYSIS}

Tables 4 and 5 shows the markers considered to be significantly associated with rust or powdery mildew resistance traits according to the threshold of 0.25 for $Q$-value in the FDR test
(Newell et al., 2012) in any of the models corrected for population structure.

As expected, considerably fewer markers showed a significant association with rust resistance when applying a correction accounting for the population structure than when using GLM alone (for simplicity GLM alone and GLM-Q-PCA are not presented in the tables). The low ability of the GLM alone to 


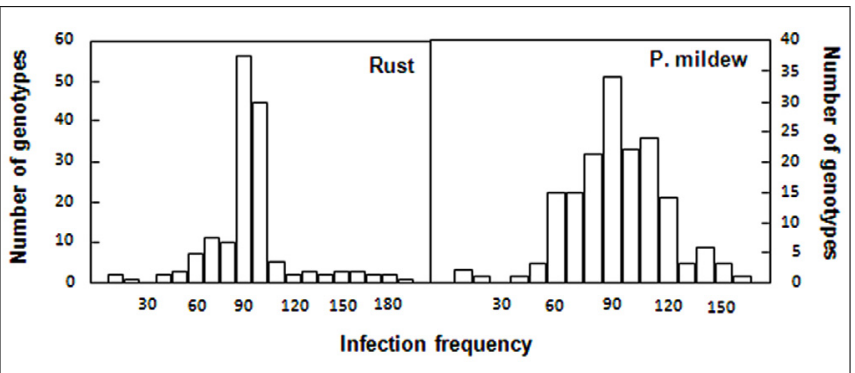

FIGURE 4 | Distribution of the infection frequency of the rust and powdery mildew infection in the oat collection. Infection frequency recorded as number of pustules per unit area for rust and percentage of leaf covered by mycelium for powdery mildew were converted into relative values, expressed as percentage of the reading of the susceptible controls, respectively.

Table 2 | Mean comparison between subpopulations for the accessions assigned to a subpopulation $(>80 \%$ membership in the subpopulation).

\begin{tabular}{lll}
\hline Subpopulation & Rust $^{\mathbf{a}}$ & $\mathbf{P M}^{\mathbf{b}}$ \\
\hline 1 & $90.1^{\mathrm{ab}}$ & $88.6^{\mathrm{a}}$ \\
2 & $108.8^{\mathrm{a}}$ & $72.9^{\mathrm{b}}$ \\
3 & $95.5^{\mathrm{ab}}$ & $90.8^{\mathrm{a}}$ \\
4 & $86.0^{\mathrm{b}}$ & $86.1^{\mathrm{a}}$ \\
\hline
\end{tabular}

a Relative infection frequency (number of pustules $\mathrm{cm}^{-2}$ ) 8 days after rust inoculation; ${ }^{b}$ PM (powdery mildew) resistance expressed as relative disease severity (percentage of the leaf covered by mycelium) 8 days after powdery mildew inoculation.

account for false positives was confirmed when the distribution of observed $p$-values was plotted in the negative $\log _{10}$ scale (Figure S2). The distribution of the $p$-values did not fit with the expected values represented by the solid line, indicating an over-abundance of low $p$-values. However, in the models accounting for structure the distribution of $p$-values fitted better with the expectation. Interestingly, population structure correction by PCA was more efficient in removing false positives than by STRUCTURE software for this data set (Figure S2). Five markers significantly associated with rust resistance were found. Markers oPt-11795 and MAMA5163 were the two most significantly associated showing significant association in all models tested including MLM and explained 20 and $10 \%$ respectively of the variation observed for this trait (Table 4). This last model showed almost a perfect fit between the observed and expected $p$-values except for few of the values, which is the characteristic of a model that sufficiently accounts for the number of false positives. Three additional markers, AM30178 , AME176-3, and oPt-15665 were significantly associated in all GLM models including those accounting for population structure (GLM-Q, GLM-PCA, and GLM Q+PCA) but not in the MLM models (Table 4).

A significant reduction of associated markers with powdery mildew resistance in oat at seedling stage was also observed after correcting for the population structure than when using GLM alone. The low efficiency of the GLM alone in this data set was confirmed by the low fit of the observed and expected distribution of the $p$-values (Figure S3). Since the significance of the oPt-14317 marker association with powdery mildew resistance in seedlings according to GLM-Q model was at the limit of 0.25 (Table 5) and since it was not highlighted by the other models it was not considered to be strongly associated. Indeed, distribution of the $p$-values in the GLM model corrected with Q indicated also a relative over-abundance of low $p$-values. Interestingly, the reverse tendency was observed in the MLM with $p$-values moving down the expected values, indicating a scarcity of low $p$ values and indicating that the GLM+PCA or GLM+Q+PCA were the best fitted models. This indicated the importance of testing the models in order to help in the selection of the most robust markers.

In order to find markers associated with powdery mildew resistance we took advantage of a previous detailed evaluation for powdery mildew adult plant resistance performed in a subpopulation of this collection (Sánchez-Martín et al., 2011). In this, following a preliminary field assessment, 54 genotypes representing the different clusters were evaluated under controlled conditions for adult plant resistance. This population covered a continuous range for powdery mildew resistance between 0 and $100 \%$, showed a similar structure to the full oat collection and showed 414,311 significant marker pairs in linkage disequilibrium with 70,657 of them showing a $r^{2}<0.1$ (Figures S1A-C). Association analysis for adult plant resistance yielded a marker, oPt-5014, highly significant in all models tested. This marker explained $\sim 30 \%$ of the observed variation according to $r^{2}$. In addition, two other markers, oPt-3306 and oPt-793335, were strongly associated in the GLM performed accounting for population structure, both through Q covariates and PCA. Distribution of the p-values in these models and particularly in that taking into account both $\mathrm{Q}$ and PCA fitted well with the expectation indicating a good consistency of the markers. Again, with this data set the MLM model

Table 3 | Statistic for stress resistance and percentage of variation of these traits explained by population structure $(K=4)$ through multiple linear regression.

\begin{tabular}{lcccccc}
\hline Trait & Mean & Minimum & Maximum & SD & CV (\%) & Variance (\%) \\
\hline Rust $^{\mathrm{a}}$ & 89.57 & 2.05 & 196.40 & 28.51 & 31.83 & $1.5^{\mathrm{ns}}$ \\
$\mathrm{PM}^{\mathrm{b}}$ & 85.51 & 0.00 & 150 & 27.44 & 32.09 & $3.7^{*}$
\end{tabular}

${ }^{a}$ Relative infection frequency (number of pustules $\mathrm{cm}^{-2}$ ) 8 days after rust inoculation; ${ }^{\mathrm{b}} \mathrm{PM}$ (powdery mildew) resistance expressed as relative disease severity (percentage of the leaf covered by mycelium) 8 days after powdery mildew inoculation. 
Table 4 | Markers associated with rust resistance according to different models: general lineal model (GLM) corrected for population structure according to percentage of admixture coefficients (Q), principal component covariates (PCA), and mixed lineal model (MLM), corrected with kinship and structure matrices.

\begin{tabular}{|c|c|c|c|c|c|c|c|c|c|}
\hline \multirow[t]{2}{*}{ Marker } & \multicolumn{3}{|c|}{ GLM+0 } & \multicolumn{3}{|c|}{ GLM+PCA } & \multicolumn{3}{|c|}{ MLM } \\
\hline & $\mathbf{p}$ & FDR & $r^{2 a}$ & $\mathbf{p}$ & FDR & $r^{2}$ & $\mathbf{p}$ & FDR & $r^{2}$ \\
\hline oPt-11795 & $1.6 \cdot 10^{-7}$ & $2.6 \cdot 10^{-4}$ & 0.18 & $3.7 \cdot 10^{-7}$ & $6.0 \cdot 10^{-4}$ & 0.16 & $1.6 \cdot 10^{-7}$ & $2.7 \cdot 10^{-4}$ & 0.22 \\
\hline MAMA5-163 & $3.2 \cdot 10^{-4}$ & 0.11 & 0.08 & $1.9 \cdot 10^{-4}$ & 0.10 & 0.08 & $1.3 \cdot 10^{-4}$ & 0.11 & 0.10 \\
\hline AM30-178 & $3.5 \cdot 10^{-5}$ & 0.02 & 0.10 & $9.4 \cdot 10^{-5}$ & 0.07 & 0.09 & $1.0 \cdot 10^{-3}$ & 0.49 & 0.07 \\
\hline AME176-3 & $4.8 \cdot 10^{-4}$ & 0.11 & 0.09 & $8.0 \cdot 10^{-4}$ & 0.25 & 0.08 & $1.2 \cdot 10^{-3}$ & 0.49 & 0.09 \\
\hline oPt-15665 & $7.0 \cdot 10^{-3}$ & 0.42 & 0.06 & $9.8 \cdot 10^{-4}$ & 0.20 & 0.08 & $2.9 \cdot 10^{-3}$ & 0.72 & 0.08 \\
\hline
\end{tabular}

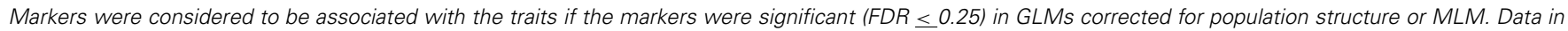
bold indicates values statistically significant according to the false discovery rate (FDR) test.

a Percentage of phenotypic variance (partial $r^{2} \times 100 \%$ ) of the total variation explained by the marker after fitting the other model effects.

Table 5 | Markers associated with powdery mildew resistance according to different models: general lineal model (GLM) corrected for population structure according to percentage of admixture coefficients (Q), principal component covariates (PCA), and mixed lineal model (MLM), corrected with kinship and structure matrices.

\begin{tabular}{|c|c|c|c|c|c|c|c|c|c|}
\hline \multirow[t]{2}{*}{ Marker } & \multicolumn{3}{|c|}{ GLM+Q } & \multicolumn{3}{|c|}{ GLM+PCA } & \multicolumn{3}{|c|}{ MLM } \\
\hline & $\mathbf{p}$ & FDR & $r^{2 a}$ & $\mathbf{p}$ & FDR & $r^{2}$ & $\mathbf{p}$ & FDR & $\mathbf{r}^{2}$ \\
\hline \multicolumn{10}{|c|}{ Seedling Stage } \\
\hline oPt-14317 & $1.5 \cdot 10^{-4}$ & 0.25 & 0.10 & $3.8 \cdot 10^{-4}$ & 0.45 & 0.09 & $8.0 \cdot 10^{-4}$ & 0.90 & 0.09 \\
\hline \multicolumn{10}{|c|}{ Adult Plant Stage } \\
\hline oPt-5014 & $7.1 \cdot 10^{-6}$ & 0.01 & 0.34 & $6.7 \cdot 10^{-6}$ & $5.4 \cdot 10^{-3}$ & 0.36 & $3.2 \cdot 10^{-4}$ & 0.19 & 0.35 \\
\hline oPt-3306 & $5.7 \cdot 10^{-5}$ & 0.04 & 0.29 & $7.5 \cdot 10^{-5}$ & 0.04 & 0.30 & $7.7 \cdot 10^{-4}$ & 0.62 & 0.30 \\
\hline oPt-793335 & $3.5 \cdot 10^{-4}$ & 0.01 & 0.24 & $5.0 \cdot 10^{-6}$ & $5.4 \cdot 10^{-3}$ & 0.36 & $2.1 \cdot 10^{-3}$ & 0.99 & 0.26 \\
\hline
\end{tabular}

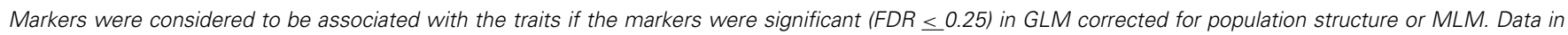
bold indicates values statistically significant according to the false discovery rate (FDR) test.

a Percentage of phenotypic variance (partial $r^{2} \times 100 \%$ ) of the total variation explained by the marker after fitting the other model effects.

seemed to be excessively restrictive in respect to the significance of the markers (Figure S4).

\section{SEOUENCE HOMOLOGY}

Although the markers significantly associated with the observed phenotypes are likely to be non-functional as they have been identified through LD, sequence information is available for many of the DArT markers evaluated (Tinker et al., 2009, Table S1) and analysis with BlastX proved interesting. Table 6 show the most significant matches found with BLAST searches (BLASTX) against public databases for associated DArT markers. For most DArT markers, no significant match to specific genic sequences was identified with most significant hits corresponding to repetitive sequences and retrotransposons (i.e., oPt-5014). However, moderately significant matches to disease resistance genes, including the wheat rust resistance locus $\operatorname{Lr} 21$, were seen for a DArT marker flanking MAMA5, oPt-14345 (FI159838, Tinker et al., 2009). In addition, the marker oPt-11795 showed homology with an autophagy-related protein 2 from Triticum urartu and marker oPt-15665 with an anthocyanin 5-aromatic acyltranferase of Aegilops tauschii.

\section{DISCUSSION}

As a first step for the association study, population structure was inferred since it has great implications on the design and analysis of GWAS. The different approaches used here indicated moderate population structure within the germplasm collection evaluated. Thus, four oat groups could be detected albeit they presented a certain degree of admixture according to STRUCTURE software with up to $30 \%$ of accessions having less than $80 \%$ membership to a determinate group. This was also observed following PCA with several accessions covering "gaps" between clusters. Interestingly the group comprising the commercial varieties showed the highest degree of admixture, most likely due to a sharing of common ancestors in their genealogy as reported by Montilla-Bascón et al. (2013). One particular concern to oats is the existence of potential population structure arising from the different oat types, winter or spring sown, or interbreeding species such as the white and red (formerly A. byzantina) oats. Indeed, in a study by Newell et al. (2012) a small cluster of red oat differentiated from the rest of the collection. Taking into account that our collection was consciously formed with diverse oat types to achieve high genetic diversity, its population structure 
Table 6 | Potential homologous sequences of significant markers using the function BlastX of the BLAST algorithm (Altschul et al., 1990).

\begin{tabular}{|c|c|c|c|c|c|c|}
\hline Marker & Blastx & Species & E-value & $\operatorname{Cov}(\%)$ & Ident(\%) & Accession number \\
\hline oPt-11795 & Autophagy-related protein 2 & Triticum urartu & $1 \mathrm{E}-12$ & 85 & 38 & EMS54055 \\
\hline \multirow[t]{2}{*}{ oPt-15665 } & Anthocyanin 5-aromatic acyltransferase & Aegilops tauschii & $1 \mathrm{E}-23$ & 54 & 56 & ЕMT29726 \\
\hline & Hypothetical protein & Sorghum bicolor & $1 \mathrm{E}-22$ & 65 & 41 & XP_002450696 \\
\hline \multirow{4}{*}{ oPt-5014 } & Hypothetical protein & S. bicolor & $2 \mathrm{E}-26$ & 90 & 56 & XP_002450843 \\
\hline & Hypothetical protein & S. bicolor & $5 \mathrm{E}-26$ & 93 & 58 & XP_002459653 \\
\hline & Uncharacterized protein & Brachypodium distachyon & $2 \mathrm{E}-25$ & 93 & 53 & XP_010233076 \\
\hline & Uncharacterized protein & B. distachyon & $1 \mathrm{E}-24$ & 98 & 47 & XP_010239298 \\
\hline
\end{tabular}

Blastx 2.2.30. Data of release January 11, 2015 1:49 A.M.

was considered relatively weak compared with that found in other cereals such as barley (Hamblin et al., 2010) or wheat (Stich et al., 2008).

Despite the relative moderate structure of this oat population, it contains a reasonably high genetic diversity, showing $\sim 10 \%$ of polymorphic markers as in the original oat panel (Tinker et al., 2009). This is an important feature in order to find markers significantly associated with a trait (Ingvarsson and Street, 2011). Estimation of genetic distances between accessions and clusters revealed inter- and intra-group genetic diversity which is confirmed by phenotypic evaluation for responses to rust and powdery mildew that showed wide variability in the degree of resistance which extended to complete susceptibility. As expected the less divergent groups were the white oat landraces adapted to high and low altitude followed by commercial cultivars while the most distant group was the red oats.

The extent of LD in a species influences the strength and resolution of GWAS. The study of linkage disequilibrium in the oat collection showed a very high number of marker pairs in significant LD. This indicated high genome coverage with non-associated markers. In oats it has been proposed that a marker every $\mathrm{cM}$ (2,000 marker in total) would explain, on average $20 \%$ of QTL variance. This is not very different from other self-pollinated cereals such as barley in which LD decay is expected to occur over relatively long map distances compared with allogamous cereals such as maize for which reduction of $r^{2}$ to 0.15 have been reported to occur within 500 bp (Tenaillon et al., 2001). Our work using 1,712 markers should cover a significant part of the genome although increasing the number and distribution of markers would increase the probability of identifying additional markers in high LD with a QTL.

The molecular marker data set in combination with phenotype evaluation was used to examine linkage-related marker-trait associations. Separating the role of population structure and genetic linkage as causes for marker-trait association remains the greatest challenge in association analysis (Achleitner et al., 2008). The five models used in this study accounted for "Q" (population structure from subpopulations) and/or "K" (genetic similarity in the background from shared kinship) which may be important to identify marker-phenotype associations not related to genetic linkage between markers and QTL. In addition models containing PCA covariates, which may account for some proportion of both "Q" and " $K$ " were also tested. A tentative comparison between the GLM and the MLM models was performed since MLM models that accounts for kinship relationships, such as that described by Yu et al. (2006), might remove more of the structure effect. This point was demonstrated by Brown et al. (2008) in sorghum and Cockram et al. (2008) in barley. In our analysis the MLM was generally excessively restrictive and did not outperform the GLMs with structure covariates. Overall, for our data set the GLM+PCA offered the best control of type I errors. Thus, co-examination of different models and traits can provide an informative summary of the major trends affecting the analysis.

Five markers, two of them found highly significant in all models tested, were associated with rust resistance. No significant similarity was identified by BLASTN or BLASTX against NCBI databases other than with putative repetitive elements or retrotransposons. However, two of these DArT sequences showed similarity to an autophagy-related protein 2 and to an anthocyanin 5-aromatic acyltransferase that have been related to the plant immune defense reaction. Thus, recently an autophagy-related protein 2 Arabidopsis mutant, atg2-2, has been reported to have enhanced resistance to powdery mildew (Wang et al., 2011) and expression of an anthocyanin 5-aromatic acyltransferase have been found to be altered in resistant $A$. thaliana ecotypes infected with cucumber mosaic virus (Ishihara et al., 2004). However, further work would be needed to ascertain the relationship between the DArTs markers and these genes.

Despite the wide distribution of powdery mildew resistance in our collection, strong association between any markers and seedling resistance was not detected. It may be possible that the combination of marker density and the phenotypic variation were insufficient. Polymorphisms causing variation for this trait may have been in linkage equilibrium with our markers, and higher marker densities could have uncovered more QTLs. Alternatively, a high number of rare alleles causing variation in seedling powdery 
mildew resistance in our collection might cause less variation in the data and therefore be undetected. Indeed, rare alleles are a leading hypothesis for the "missing heritability observation" in human association studies (Yang et al., 2010). The low association for this trait could also be due to the development of markers from a genetically narrow set of germplasm in relation to the lines used in this study. However, this is highly unlikely since DArT markers were developed from a panel of 60 accessions of global representation. Interestingly one DArT sequence, oPt-5014, was strongly associated with powdery mildew resistance in adult plants. The strong association observed taking into account the relative low number of accessions evaluated for this trait, suggest that a careful selection of accessions covering a complete range of phenotypic and genotypic variation may be adequate in some cases to find significant associations. Marker oPt-5014 was associated with hypothetical proteins of sorghum, wheat and rice containing a Zinc knuckle domain (pfam14392) which has been detected in several plants transcription factors and might therefore be involved in the regulation of gene expression.

Recent oat maps sharing common markers allow us to locate the DArT markers more specifically within the oat genome (Figure S5). According to Tinker et al. (2009), oPt-11795 marker maps onto $\mathrm{KO} 32$ which is equivalent to chromosome $4 \mathrm{C}$ in the first physically anchored consensus oat map (Oliver et al., 2011) where there are no previously reported crown rust resistance genes. Recent studies showed synteny between this chromosome and Brachypodium distachyon chromosome 4, Oryza sativa chromosome 9 and wheat chromosome 5BL where regions controlling disease resistance have been described; QTLs for resistance to the rust fungus Puccinia brachypodii have been reported in chromosome 4 of B. distachyon (Barbieri et al., 2012), a powdery mildew resistance gene PmAS846 mapped in wheat chromosome 5BL (Xue et al., 2012) and a locus associated with broad-spectrum resistance to rice blast, $\mathrm{Pi5}(\mathrm{t})$, mapped onto rice chromosome 9. MAMA5 is reported by Wight et al. (2003) to map near to the marker cdo53 on KO17 equivalent to chromosome 9D (Oliver et al., 2013). Interestingly, the partial crown rust resistance Pc38 that cluster with Pc62 and Pc63 (Harder et al., 1980) also maps in this position (Wight et al., 2004) together with the major QTL for partial rust resistance, Prq1b (Portyanko et al., 2005). AME176 maps onto chromosome 15A (unpublished Buffalo $\times$ Tardis results) which shows homology with chromosome 9D where according to Oliver et al. (2013) a number of other resistance genes map. According to Tinker et al. (2009), oPt-14317 maps onto KO22_44_18 within the same framework marker as AM102 now annotated as chromosome 19A. This is a similar position to where the dominant powdery mildew resistance gene Eg5 has been mapped (Yu and Herrmann, 2006). Finally, oPt-5014 has been mapped in a number of populations (e.g., Hizbai et al., 2012; He et al., 2013) onto chromosome 21D. This chromosome is also known to contain a number of crown rust resistance genes such as Pc54, Pc59, and Pc68. However, lack of common markers makes it difficult to determine how close oPt-5014 is to these genes.

Overall, the markers showing the strongest association in this study provide ideal candidates for further studies and future inclusion in strategies of MAS.

\section{AUTHOR CONTRIBUTIONS}

GM-B, NR, and JS-M carried out most of the experimental work and data analysis. DR contributed to the disease resistance aspects. $\mathrm{TL}, \mathrm{CH}$, and LM contributed to the genetic aspects. EP designed experiments, and contributed to the interpretation of results and writing of the manuscript. NR, DR, TL, CH, and LM also contributed to critical reading and writing.

\section{ACKNOWLEDGMENTS}

This work was supported by the Spanish Ministry of Economy and Competitivity AGL2013-48687-R, the European Regional Development Funds, a JAE PreDoc fellowship from CSIC to GM-B, a Ramon y Cajal funds from CSIC to NR and an Institute Strategic Programme Grant University (BBS/E/W/10962A01) to IBERS, Aberystwyth awarded by the Biotechnology and Biological Sciences Research Council (BBSRC), UK. We thank CRF (INIA, Madrid) for kindly supplying the seeds of the accessions used.

\section{SUPPLEMENTARY MATERIAL}

The Supplementary Material for this article can be found online at: http://www.frontiersin.org/journal/10.3389/fpls.2015.00103/ abstract

\section{REFERENCES}

Achleitner, A., Tinker, N. A., Zechner, E., and Buerstmayr, H. (2008). Genetic diversity among oat varieties of worldwide origin and associations of AFLP markers with quantitative traits. Theor. Appl. Genet. 117, 1041-1053. doi: 10.1007/s00122-008-0843-y

Altschul, S. F., Gish, W., Miller, W., Myers, E. W., and Lipman, D. J. (1990). Basic local aligment search tool. J. Mol. Biol. 215, 403-410.

Asoro, F. G., Newell, M. A., Scott, M. P., Beavis, W. D., and Jannink, J. L. (2013). Genome-wide association study for beta-glucan concentration in elite North American oat. Crop Sci. 53, 542-553. doi: 10.2135/cropsci2012. 01.0039

Barbieri, M., Marcel, T. C., Niks, R. E., Francia, E., Pasquariello, M., Mazzamurro, V., et al. (2012). QTLs for resistance to the false brome rust Puccinia brachypodii in the model grass Brachypodium distachyon L. Genome 55, 152-163. doi: $10.1139 / g 2012-001$

Benjamini, Y., and Hochberg, Y. (1995). Controlling the false discovery rate: a practical and powerful approach to multiple testing. J. R. Statist. Soc. B 57, 289300.

Bradbury, P. J., Zhang, Z., Kroon, D. E., Casstevens, T. M., Ramdos, Y., and Buckle, E. S. (2007). TASSEL: software for association mapping of complex traits in diverse samples. Bioinformatics 23, 2633-2635. doi: 10.1093/bioinformatics/ btm308

Breseghello, F., and Sorrells, M. E. (2006). Association analysis as a strategy for improvement of quantitative traits in plants. Crop Sci. 46, 1323-1330. doi: 10.2135/cropsci2005.09-0305

Brown, P. J., Rooney, W. L., Franks, C., and Kresovich, S. (2008). Efficient mapping of plant height quantitative trait loci in a sorghum association population with introgressed dwarfing genes. Genetics 180, 629-637. doi: 10.1534/genetics.108.092239

Cockram, J., White, J., Leigh, F. J., Lea, V. J., Chiapparin, E., Laurie, D. A., et al. (2008). Association mapping of partitioning loci in barley. BMC Genet. 9:16. doi: 10.1186/1471-2156-9-16

Cordell, H. J., Bentham, J., Topf, A., Zelenika, D., Heath, S., Mamasoula, C., et al. (2013). Genome-wide association study of multiple congenital heart disease phenotypes identifies a susceptibility locus for atrial septal defect at chromosome 4p16. Nat. Genet. 45, 822-824. doi: 10.1038/ng.2637

de Alencar Figueiredo, L. F., Sine, B., Chantereau, J., Mestres, C., Fliedel, G., Rami, J. F., et al. (2010). Variability of grain quality in sorghum: association with polymorphism in Sh2, Bt2, SssI, Ae1, Wx and O2. Theor. Appl. Genet. 121, 1171-1185. doi: $10.1007 /$ s00122-010-1380-z 
Evanno, G., Regnaut, S., and Goudet, J. (2005). Detecting the number of clusters of individuals using the software STRUCTURE: a simulation study. Mol. Ecol. 14, 2611-2620. doi: 10.1111/j.1365-294X.2005.02553.x

Falconer, D. S., and MacKay, T. F. C. (1996). Introduction to Quantitative Genetics, 4th Edn. Essex: Longman Scientific \& Technical.

Flint-Garcia, S. A., Thornsberry, J. M., and Buckler, E. S. (2003). Structure of linkage disequilibrium in plants. Ann. Rev. Plant Biol. 54, 357-374. doi: 10.1146/annurev.arplant.54.031902.134907

Gaut, B. S., and Long, A. D. (2003). The lowdown on linkage disequilibrium. Plant Cell 15, 1502-1506. doi: 10.1105/tpc.150730

Gupta, P. K., Rustgi, S., and Kulwal, P. L. (2005). Linkage disequilibrium and association studies in higher plants: present status and future prospects. Plant Mol. Biol. 57, 461-485. doi: 10.1007/s11103-005-0257-z

Gurung, S., Mamidi, S., Bonman, J. M., Xiong, M., Brown-Guedira, G., and Adhikari, T. B. (2014). Genome-wide association study reveals novel quantitative trait loci associated with resistance to multiple leaf spot diseases of spring wheat. PLoS ONE 9:e108179. doi: 10.1371/journal.pone.0108179

Hamblin, M. T., Close, T. J., Bhat, P. R., Chao, S., Kling, J. G., Abrahamm, K. J., et al. (2010). Population structure and linkage disequilibrium in us barley germplasm: implications for association mapping. Crop Sci. 50, 556-566. doi: 10.2135/cropsci2009.04.0198

Hammer, O., Harper, D., and Ryan, P. (2001). PAST: paleontological statistics software for education and data analysis. Paleontol Electron 4, 1-9.

Harder, D. E., McKenzie, R. I. H., and Martens, J. W. (1980). Inheritance of crown rust resistance in 3 accesions of Avena sterilis. Can. J. Genet. Cytol. 22, 27-33. doi: 10.1139/g80-005

He, X., Skinnes, H., Oliver, R. E., Jackson, E. W., and Bjornstad, A. (2013). Linkage mapping and identification of QTL affecting deoxynivalenol (DON) content (Fusarium resistance) in oats (Avena sativa L.). Theor. Appl. Genet. 126, 2655-2670. doi: 10.1007/s00122-013-2163-0

Hizbai, B. T., Gardner, K. M., Wight, C. P., Dhanda, R. K., Molnar, S. J., Johnson, D., et al. (2012). Quantitative trait loci affecting oil content, oil composition, and other agronomically important traits in oat. Plant Genome 5, 164-175. doi: 10.3835/plantgenome2012.07.0015

Huang, X., Wei, X., Sang, T., Zhao, Q., Feng, Q., Zhao, Y., et al. (2010). Genomewide association studies of 14 agronomic traits in rice landraces. Nat. Genet. 42, 961-967. doi: 10.1038/ng.695

Ihaka, R., and Gentleman, R. (1996). R: a language for data analysis and graphics. J. Comput. Graph Statist. 5, 299-314.

Ingvarsson, P. K., and Street, N. R. (2011). Association genetics of complex traits in plants. New Phytol. 189, 909-922. doi: 10.1111/j.1469-8137.2010.03593.x

Ishihara, T., Sakurai, N., Sekine, K. T., Hase, S., Ikegami, M., Shibata, D., et al. (2004). Comparative analysis of expressed sequence tags in resistant and susceptible ecotypes of Arabidopsis thaliana infected with cucumber mosaic virus. Plant Cell Physiol. 45, 470-480. doi: 10.1093/pcp/pch057

Jackson, E. W., Obert, D. E., Avant, J. B., Harrison, S. A., Chong, J., Carson, M. L. et al. (2010). Quantitative trait loci in the Ogle/TAM O-301 oat mapping population controlling resistance to Puccinia coronata in the field. Phytopathology 100 484-492. doi: 10.1094/PHYTO-100-5-0484

Jackson, E. W., Wise, M., Bonman, J. M., Obert, D. E., Hu, G., and Peterson, D. M. (2008). QTLs affecting alpha-tocotrienol, alpha-tocopherol, and total tocopherol concentrations detected in the Ogle/TAM O-301 oat mapping population. Crop Sci. 48, 2141-2152. doi: 10.2135/cropsci2007.11.0658

Jannink, J. L., Bink, M., and Jansen, R. C. (2001). Using complex plant pedigrees to map valuable genes. Trends Plant Sci. 6, 337-342. doi: 10.1016/S1360 1385(01)02017-9

Jia, G., Huang, X., Zhi, H., Zhao, Y., Zhao, Q., Li, W., et al. (2013). A haplotype map of genomic variations and genome-wide association studies of agronomic traits in foxtail millet (Setaria italica). Nat. Genet. 45, 957-961. doi: 10.1038/ng.2673

Klein, R. J., Zeiss, C., Chew, E. Y., Tsai, J. Y., Sackler, R. S., Haynes, C., et al. (2005). Complement factor $\mathrm{H}$ polymorphism in age-related macular degeneration. Science 308, 385-389. doi: 10.1126/science.1109557

Kraakman, A. T. W., Niks, R. E., Van den Berg, P., Stam, P., and Van Eeuwijk, F. A. (2004). Linkage disequilibrium mapping of yield and yield stability in modern spring barley cultivars. Genetics 168, 435-446. doi: 10.1534/genetics.104. 026831

Kump, K. L., Bradbury, P. J., Wisser, R. J., Buckler, E. S., Belcher, A. R., OropezaRosasand, M. A., et al. (2011). Genome-wide association study of quantitative resistance to southern leaf blight in the maize nested association mapping population. Nat. Genet. 43, 163-168. doi: 10.1038/ng.747

Lee, J. Y., Lee, B. S., Shin, D. J., Woo Park, K., Shin, Y. A., Joong, K. K., et al. (2013). A genome-wide association study of a coronary artery disease risk variant. J. Hum. Genet. 58, 120-126. doi: 10.1038/jhg.2012.124

Li, H., Peng, Z., Yang, X., Wang, W., Fu, J., Wang, J., et al. (2013). Genome-wide association study dissects the genetic architecture of oil biosynthesis in maize kernels. Nat. Genet. 45, 43-50. doi: 10.1038/ng.2484

Lyngkjær, M. F., Carver, T. L. W., and Zeyen, R. J. (1997). Suppression of resistance to Erysiphe graminis f. sp. hordei conferred by the mlo5 barley powdery mildew resistance gene. Physiol. Mol. Plant Pathol. 50, 17-36. doi: 10.1006/pmpp.1996.0068

Maloney, P. V., Lyerly, J. H., Wooten, D. R., Anderson, J. M., Livingston, D. P., Brown-Guedira, G., et al. (2011). Marker development and quantitative trait loci in a fall-sown oat recombinant inbred population. Crop Sci. 51, 490-502. doi: 10.2135/cropsci2010.04.0224

Martínez, F., Sillero, J. C., and Rubiales, D. (2007). Resistance to leaf rust in cultivars of bread wheat and durum wheat grown in Spain. Plant Breed 126, 13-18. doi: 10.1111/j.1439-0523.2007.01287.x

Miyagawa, T., Nishida, N., Ohashi, J., Kimura, R., Fujimoto, A., Kawashima, M., et al. (2008). Appropriate data cleaning methods for genome-wide association study. J. Hum. Genet. 53, 886-893. doi: 10.1007/s10038-008-0322-y

Montilla-Bascón, G., Sánchez-Martín, J., Rispail, N., Rubiales, D., Mur, L. A. J., Langdon, T., et al. (2013). Genetic diversity and population structure among oat cultivars and landraces. Plant Mol. Biol. Rep. 31, 1305-1314. doi: 10.1007/s11105013-0598-8

Morris, G. P., Ramub, P., Deshpandeb, S. P., Hashc, C. T., Shah, T., Upadhyaya, H. D., et al. (2013). Population genomic and genome-wide association studies of agroclimatic traits in sorghum. Proc. Nat. Acad. Sci. U.S.A. 110, 453-458. doi: 10.1073/pnas.1215985110

Nei, M., and Li, W. H. (1979). Mathematical model for studying genetic variation in terms of restriction endonucleasis. Proc. Nat. Acad. Sci. U.S.A. 76, 5269-5273. doi: 10.1073/pnas.76.10.5269

Newell, M. A., Asoro, F. G., Scott, M. P., White, P. J., Beavis, W. D., and Jannink, J. L. (2012). Genome-wide association study for oat (Avena sativa L.) beta-glucan concentration using germplasm of worldwide origin. Theor. Appl. Genet. 125, 1687-1696. doi: 10.1007/s00122-012-1945-0

Newell, M. A., Cook, D., Tinker, N. A., and Jannink, J. L. (2011). Population structure and linkage disequilibrium in oat (Avena sativa L.): implications for genome-wide association studies. Theor. Appl. Genet. 122, 623-632. doi: 10.1007/s00122-0101474-7

Oliver, R. E., Lazo, G. R., Lutz, J. D., Rubenfield, M. J., Tinker, N. A., Anderson, J. M., et al. (2011). Model SNP development for complex genomes based on hexaploid oat using high-throughput 454 sequencing technology. BMC Genomics 12:77. doi: 10.1186/1471-2164-12-77

Oliver, R. E., Tinker, N. A., Lazo, G. R., Chao, S., Jellen, E. N., Carson, M. L., et al. (2013). SNP discovery and chromosome anchoring provide the first physicallyanchored hexaploid oat map and reveal synteny with model species. PLoS ONE 8:e58068. doi: 10.1371/journal.pone.0058068

Portyanko, V. A., Chen, G., Rines, H. W., Phillips, R. L., Leonard, K. J., Ochocki, G. E., et al. (2005). Quantitative trait loci for partial resistance to crown rust, Puccinia coronata, in cultivated oat, Avena sativa L. Theor. Appl. Genet. 112, 195-197. doi: 10.1007/s00122-005-0072-6

Prats, E., Rubiales, D., and Jorrin, J. (2002). Acibenzolar-S-methyl-induced resistance to sunflower rust (Puccinia helianthi) is associated with an enhancement of coumarins on foliar surface. Physiol. Mol. Plant Pathol. 60, 155-162. doi: 10.1006/pmpp.2002.0385

Pritchard, J. K., Stephens, M., Rosenberg, N. A., and Donnelly, P. (2000). Association mapping in structured populations. Am. J. Hum. Genet. 67, 170-181. doi: 10.1086/302959

Rubiales, D., Brown, J. K. M., and Martín, A. (1993). Hordeum chilense resistance to powdery mildew and its potential use in cereal breeding. Euphytica 67, 215-220. doi: 10.1007/BF00040623

Sánchez-Martín, J., Rubiales, D., and Prats, E. (2011). Resistance to powdery mildew (Blumeria graminis f.sp avenae) in oat seedlings and adult plants. Plant Pathol. 60, 846-856. doi: 10.1111/j.1365-3059.2011.02453.x

Sánchez-Martín, J., Rubiales, D., Sillero, J. C., and Prats, E. (2012). Identification and characterization of sources of resistance in Avena sativa, A. byzantina and 
A. strigosa germplasm against a pathotype of Puccinia coronata f. sp. avenae with virulence against the Pc94 resistance gene. Plant Pathol. 61, 315-322. doi: 10.1111/j.1365-3059.2011.02514.x

Snowdon, R. J., and Friedt, W. (2004). Molecular markers in Brassica oilseed breeding: current status and future possibilities. Plant Breed. 123, 1-8. doi: 10.1111/j.1439-0523.2003.00968.x

Stevens, E. J., Armstrong, K. W., Bezar, H. J., and Griffin, W. B. (2004). "Fodder oats an overview," in Fodder Oats: A World Overview, eds J. M. Suttie and S. G. Reynolds (Rome: Food and Agriculture Organization of the United Nations), 1-9. Stich, B., Piepho, H.-P., Schulz, B., and Melchinger, A. E. (2008). Multi-trait association mapping in sugar beet (Beta vulgaris L.). Theor. Appl. Genet. 117, 947-954. doi: 10.1007/s00122-008-0834-z

Storey, J. D. (2002). A direct approach to false discovery rates. J. R. Statist. Soc. Ser. B 64, 479-498. doi: 10.1111/1467-9868.00346

Stracke, S., Haseneyer, G., Veyrieras, J. B., Geiger, H. H., Sauer, S., Graner, A., et al. (2009). Association mapping reveals gene action and interactions in the determination of flowering time in barley. Theor. Appl. Genet. 118, 259-273. doi 10.1007/s00122-008-0896-y

Tenaillon, M. I., Sawkins, M. C., Long, A. D., Gaut, R. L., Doebley, J. F., and Gaut, B. S. (2001). Patterns of DNA sequence polymorphisms along chromosome 1 of maize (Zea mays ssp. mays L.). Proc. Nat. Acad. Sci. U.S.A. 98, 9161-9166. doi: 10.1073/pnas. 151244298

Tinker, N. A., Kilian, A., Wight, C. P., Heller-Uszynska, K., Wenzl, P., Rines, H. W., et al. (2009). New DArT markers for oat provide enhanced map coverage and global germplasm characterization. BMC Genomics 10:39. doi: 10.1186/14712164-10-39

Wang, C., Yang, Y., Yuan, X., Xu, Q., Feng, Y., Yu, H., et al. (2014). Genome-wide association study of blast resistance in indica rice. BMC Plant Biol 14:311. doi: 10.1186/s12870-014-0311-6

Wang, Y., Nishimura, M. T., Zhao, T., and Tang, D. (2011). ATG2, an autophagy-related protein, negatively affects powdery mildew resistance and mildew-induced cell death in Arabidopsis. Plant J. 68, 74-87. doi: 10.1111/j.1365313X.2011.04669.x

Wight, C. P., O’Donoughue, L. S., Chong, J., Tinker, N. A., and Molnar, S. J. (2004). Discovery, localization, and sequence characterization of molecular markers for the crown rust resistance genes Pc38, Pc39, and Pc48 in cultivated oat (Avena sativa L.). Mol. Breed 14, 349-361. doi: 10.1007/s11032-004-0148-Z

Wight, C. P., Tinker, N. A., Kianian, S. F., Sorrells, M. E., O’Donoughue, L. S., Hoffman, D. L., et al. (2003). A molecular marker map in 'Kanota’ x 'Ogle’ hexaploid oat (Avena spp.) enhanced by additional markers and a robust framework. Genome 46, 28-47. doi: 10.1139/g02-099
Xue, F., Wang, C., Li, C., Duan, X., Zhou, Y., Zhao, N., et al. (2012). Molecular mapping of a powdery mildew resistance gene in common wheat landrace Baihulu and its allelism with Pm24. Theor. Appl. Genet. 125, 1425-1432. doi: 10.1007/s00122-012-1923-6

Yang, J., Benyamin, B., McEvoy, B. P., Gordon, S., Henders, A. K., Nyholt, D. R., et al. (2010). Common SNPs explain a large proportion of the heritability for human height. Nat. Genet. 42, 565-569. doi: 10.1038/ng.608

Yu, J., and Herrmann, M. (2006). Inheritance and mapping of a powdery mildew resistance gene introgressed from Avena macrostachya in cultivated oat. Theor Appl. Genet. 113, 429-437. doi: 10.1007/s00122-006-0308-0

Yu, J. M., Pressoir, G., Briggs, W. H., Bi, I. V., Yamasaki, M., Doebley, J. F., et al. (2006). A unified mixed-model method for association mapping that accounts for multiple levels of relatedness. Nat. Genet. 38, 203-208. doi: 10.1038/ng1702

Zhu, C., Gore, M., Buckler, E. S., and Yu, J. (2008). Status and prospects of association mapping in plants. Plant Genome 1, 5-20. doi: 10.3835/plantgenome2008.02.0089

Zhu, S., Rossnagel, B. G., and Kaeppler, H. F. (2004). Genetic analysis of quantitative trait loci for groat protein and oil content in oat. Crop Sci. 44, 254-260. doi: $10.2135 /$ cropsci2004.0254

Zondervan, K. T., and Cardon, L. R. (2004). The complex interplay among factors that influence allelic association. Nat. Rev. Genet. 5, 89-100. doi: 10.1038/nrg1270

Conflict of Interest Statement: The authors declare that the research was conducted in the absence of any commercial or financial relationships that could be construed as a potential conflict of interest.

Received: 23 October 2014; accepted: 08 February 2015; published online: 05 March 2015.

Citation: Montilla-Bascón G, Rispail N, Sánchez-Martín J, Rubiales D, Mur LAJ, Langdon T, Howarth CJ and Prats E (2015) Genome-wide association study for crown rust (Puccinia coronata $f$. sp. avenae) and powdery mildew (Blumeria graminis $f$. sp. avenae) resistance in an oat (Avena sativa) collection of commercial varieties and landraces. Front. Plant Sci. 6:103. doi: 10.3389/fpls.2015.00103

This article was submitted to Crop Science and Horticulture, a section of the journal Frontiers in Plant Science.

Copyright (C) 2015 Montilla-Bascón, Rispail, Sánchez-Martín, Rubiales, Mur, Langdon, Howarth and Prats. This is an open-access article distributed under the terms of the Creative Commons Attribution License (CC BY). The use, distribution or reproduction in other forums is permitted, provided the original author(s) or licensor are credited and that the original publication in this journal is cited, in accordance with accepted academic practice. No use, distribution or reproduction is permitted which does not comply with these terms. 